\title{
Dominant Atrial Fibrillatory Frequency Estimation using an Extended Kalman Smoother
}

\author{
Ebadollah Kheirati Roonizi, Roberto Sassi \\ Dipartimento di Informatica, Università degli Studi di Milano, Italy
}

\begin{abstract}
In patients with atrial fibrillation $(A F)$, the dominant repetition rate of the atrial fibrillatory waves (f-waves), or fibrillatory frequency $(F F)$, (usually in the range 3-12 $\mathrm{Hz}$ ) plays an important role for non-invasive assessment of atrial electrical remodeling. It is usually assessed from the electrocardiogram (ECG) by signal processing tools such as power spectral analysis and short-time Fourier transform (STFT), after ventricular activity (VA) cancellation.

FF can also be estimated simultaneously with VA detection using an extended Kalman smoother (EKS), as recently proposed by us. In this paper, we try to simplify the model and adapt it to situations in which less computational power is available and only short signals are considered (e.g., mobile or E-health applications). In the proposed model, the ventricular activity (VA) is represented by a sum of Gaussian kernels, while a single sinusoidal function with constant frequency is employed for the atrial activity (AA). The strategy was validated using 290 synthetic signals obtained from ECGs in sinus rhythm (Physionet PTBDB), where P-waves were replaced by artificial $f$-waves, at different signal-to-noise (SNR) ratios. At a SNR of 0,20 and $40 d B$, the average root mean square errors were $0.22,0.08$ and $0.01 \mathrm{~Hz}$ respectively.
\end{abstract}

\section{Introduction}

In patients with atrial fibrillation (AF), the dominant repetition rate of the atrial fibrillatory waves (f-waves), or fibrillatory frequency (FF, usually in the range 3-12 $\mathrm{Hz}$ ) plays an important role for non-invasive assessment of atrial electrical remodeling. It can be assessed from the electrocardiogram (ECG) by signal processing tools such as power spectral analysis and short-time Fourier transform (STFT), after atrial activity (AA) isolation. However, atrial and ventricular activities (VA) overlap in both time and frequency. As a result, the basic problem is to extract the f-waves from the mixture of ventricular (QRS-T) and atrial signals, where the interfering ventricular signals are much stronger. Hence most of the existing procedures deal with the cancellation of QRS-T, instead of directly with extraction of f-waves.
The current methods for VA cancellation are based on single, (e.g. average beat subtraction, ABS [1], adaptive singular value decomposition, SVD [2], wavelet transform [3], maximum likelihood estimation, MLE [4]) or multichannel recordings (e.g. spatiotemporal QRS-T cancellation [1], blind source separation, BSS [5,6], and principal component analysis, PCA [7]). FF estimation simultaneously with VA detection has been recently proposed in [8]. The goal of this paper is to simplify the model proposed in [8] and adapt it to situations in which there is less computational power at disposal, and only short signals are of interest (e.g., mobile or e-health applications).

\section{Method}

\subsection{Extended Kalman Filter}

Lets consider the following dynamical system with hidden state vector $x_{k}$ and observation vector $y_{k}$

$$
\left\{\begin{array}{l}
x_{k+1}=f\left(x_{k}, w_{k}, k\right) \\
y_{k}=g\left(x_{k}, v_{k}, k\right)
\end{array},\right.
$$

where $f(\cdot)$ is the evolution state function, $g(\cdot)$ represents the relationship between the hidden state and observations, $w_{k}$ and $v_{k}$ are process noise and measurement noise, with the corresponding covariance matrices $Q_{k}=E\left\{w_{k} w_{k}^{T}\right\}$ and $R_{k}=E\left\{v_{k} v_{k}^{T}\right\}$. The extended Kalman filter (EKF) is a nonlinear extended version of the well-known Kalman filter (KF) to estimate the hidden states of (1). An extended Kalman Smoother (EKS), which consists of a forward EKF stage followed by a backward recursive smoothing stage, can be employed after EKF for smoother results. In fact, it provides better estimates of the current states since it uses information brought by "future" observations.

\subsection{Normal ECG modelling}

A normal ECG beat can be represented as a linear combination of Gaussian functions with different amplitudes and widths centered at specific points in time $[9,10]$ :

$$
z=\sum_{i \in\{\mathrm{P}, \mathrm{Q}, \mathrm{R}, \mathrm{S}, \mathrm{T}\}} \alpha_{i} \exp \left[-\frac{\left(t-\theta_{i}\right)^{2}}{2 b_{i}^{2}}\right] .
$$




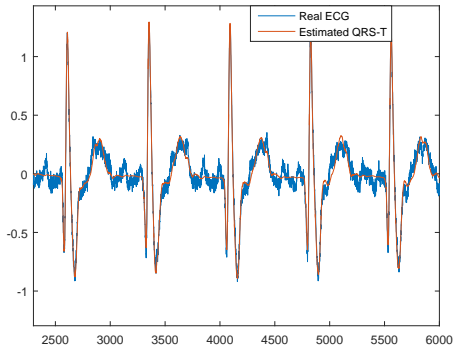

(a)QRS-T extraction provided by EKF

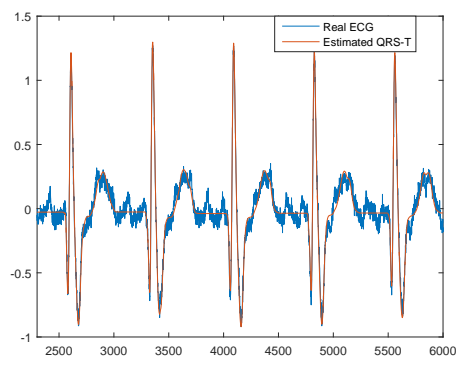

(d)QRS-T extraction provided by EKS

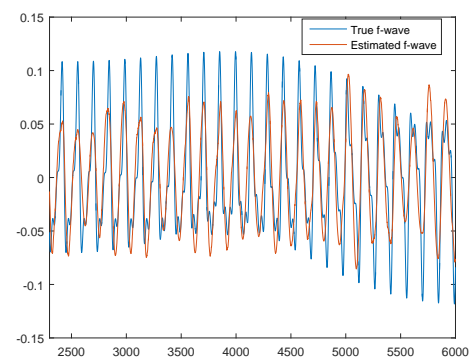

(b)f-wave extraction provided by EKF

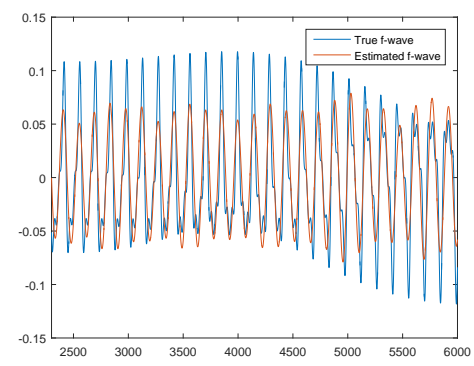

(e)f-wave extraction provided by EKS

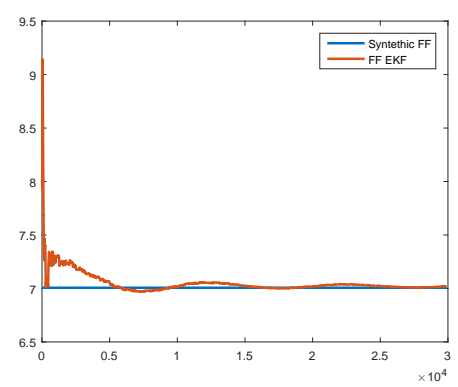

(c)FF estimated by EKF

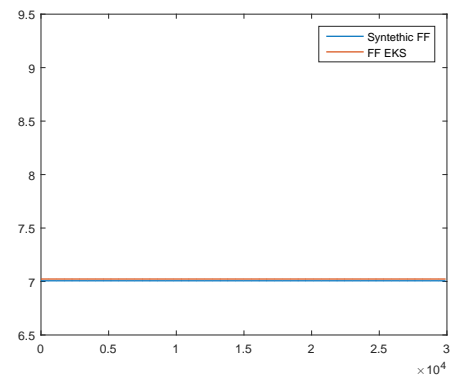

(f)FF estimated by EKS

Figure 1. AA, VA and FF estimation using EKF and EKS for a typical ECG signal.

Eq. (2) can be coupled with a model of the heart rate dynamics, to synthesize multiple ECG beats with arbitrary heart rates [9]:

$$
\left\{\begin{array}{l}
\dot{\theta}=\omega \\
\dot{z}=-\sum_{i \in\{\mathrm{P}, \mathrm{Q}, \mathrm{R}, \mathrm{S}, \mathrm{T}\}} \alpha_{i} \omega \frac{\theta-\theta_{i}}{b_{i}^{2}} \exp \left[-\frac{\left(\theta-\theta_{i}\right)^{2}}{2 b_{i}^{2}}\right]
\end{array}\right.
$$

where $\omega$ is the angular velocity of the trajectory as it moves around the limit cycle, $\theta$ is the cardiac phase, which is defined between $-\pi$ and $\pi, \alpha_{i}, b_{i}$ and $\theta_{i}$ adapt amplitude, width and center of the Gaussian kernels to the different ECG morphologies.

\subsection{ECG modelling during AF}

During AF, the $\mathrm{P}$ wave is absent and replaced by a sort of fluctuation of the baseline. Hence, we speculated that the ECG, on any lead, can be described by sum of Gaussian functions plus a sinusoidal model with a fundamental frequency and its harmonics [8]. In this work, we try to simplify further the model considering a single sinusoid for AA description. A possible discrete time model for
ECG signal $\left(s_{k}\right)$ during AF is then of the form:

$$
\left\{\begin{array}{l}
\theta_{k+1}=\left(\theta_{k}+\omega \delta\right) \quad \bmod 2 \pi \\
s_{k}=\sum_{i \in\{\mathrm{Q}, \mathrm{R}, \mathrm{S}, \mathrm{T}\}} \alpha_{i} \exp \left[-\frac{\left(\theta_{k}-\theta_{i}\right)^{2}}{2 b_{i}^{2}}\right]+p \cos \left(w_{c} k+\varphi\right)
\end{array}\right.
$$

where $f_{s}$ is the sampling rate, $w_{c}=2 \pi f_{c} / f_{s}, p$ and $\varphi$ are respectively the frequency, amplitude and phase of the sinusoid. In order to use the model with Kalman filter and track AA, VA as well as the frequency of the sinusoid, we recast (3) into:

Process equation:

$$
\left\{\begin{array}{l}
\theta_{k+1}=\left(\theta_{k}+\omega \delta\right) \quad \bmod 2 \pi \\
w_{c, k+1}=w_{c, k} \\
z_{k+1}=z_{k}-\omega \delta \sum_{i \in\{\mathrm{Q}, \mathrm{R}, \mathrm{S}, \mathrm{T}\}} \alpha_{i} \frac{\theta_{k}-\theta_{i}}{b_{i}^{2}} \exp \left[-\frac{\left(\theta_{k}-\theta_{i}\right)^{2}}{2 b_{i}^{2}}\right] \\
\phi_{k+1}-2 \cos \left(n w_{c, k}\right) \phi_{k}+\phi_{k-1}=\eta_{\phi}
\end{array}\right.
$$

Observation equation:

$$
\left\{\begin{array}{l}
\psi_{k}=\theta_{k}+v_{1, k} \\
s_{k}=z_{k}+\phi_{k}+v_{2, k}
\end{array}\right.
$$

where $\delta=1 / f_{s}$ is the sampling interval, $s_{k}$ is the real ECG and $\psi_{k}$ is the noisy cardiac phase at time instant $k . \eta_{\phi}$ is 


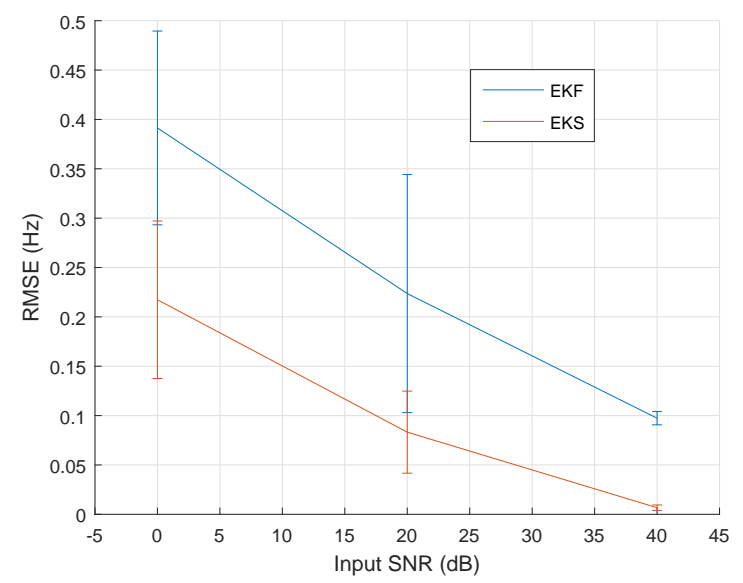

Figure 2. Mean and standard deviation of RMSE in frequency estimation for constant frequency by means of EKF and EKS, as a function of the broadband noise contaminating the input signal.

a i.i.d. Gaussian random variable representing the possible model errors, changes of fundamental frequency and phase. Finally $v_{1}$ and $v_{2}$ are zero mean random variables considered to be observation noise.

\subsection{Bayesian Filtering Framework}

The new ECG model can be utilized in a Bayesian framework for constant FF tracking, VA and AA separation. Considering the process and observation equations (4) and (5), the state variables vector, $x_{k}$, the observation vector, $y_{k}$, the process noise vector, $w_{k}$, and the observation noise vector, $v_{k}$, are defined as follows:

$$
\begin{aligned}
& x_{k}=\left[\theta_{k}, w_{c, k}, z_{k}, \phi_{k-1}, \phi_{k}\right]^{T} \\
& y_{k}=\left[\psi_{k}, s_{k}\right] \\
& w_{k}=\left[\alpha_{i}, b_{i}, \theta_{i}, \eta_{\phi}, \omega\right] \\
& v_{k}=\left[v_{1_{k}}, v_{2_{k}}\right]
\end{aligned}
$$

with the corresponding process noise and measurement noise covariance matrices $Q_{k}=E\left\{w_{k} w_{k}^{T}\right\}$ and $R_{k}=$ $E\left\{v_{k} v_{k}^{T}\right\}$.

\subsection{AF synthetic signals}

We implemented the proposed Bayesian framework and applied it on synthetic signals taken from the PhysioNet PTB Diagnostic ECG Database [11] (sampling frequency: $1000 \mathrm{~Hz}$; resolution: 16-bit). $290 \mathrm{ECG}$ segments (one from each subject), with duration of $30 \mathrm{~s}$, were selected from each of the twelve conventional leads, at the beginning of the recording. P-waves were separated and removed using the approach proposed for ECG components separation in [12]. Then synthetic f-waves were generated using a sawtooth model introduced in $[1,13]$, and added to the ECG segments to study the performance of the proposed filter. The synthetic f-wave model is described by a fundamental components and $M-1$ of its harmonics

$$
\xi_{d, k}=\sum_{m=1}^{M} a_{m, k} \sin \left[m \omega_{0} k+\frac{\Delta_{f}}{f_{f}} \sin \left(\omega_{f} k\right)\right],
$$

where the fundamental frequency $\omega_{0}=2 \pi f_{0}$ has the maximum deviation $\Delta_{f}$ and the modulation frequency $\omega_{f}=$ $2 \pi f_{f}$. Furthermore the amplitude $a_{m, k}$ is defined so that a signal with sawtooth characteristic is produced

$$
a_{m, k}=\frac{2}{m \pi}\left[a+\Delta a \sin \left(\omega_{a} k\right)\right],
$$

where $a$ denotes the sawtooth amplitude, $\Delta a$ the modulation peak amplitude and $\omega_{a}=2 \pi f_{a}$ is the amplitude modulation frequency. In our simulations, we selected: $M=3, \Delta_{f} \approx 0.25 \mathrm{~Hz}, f_{f} \approx 0.2 \mathrm{~Hz}, f_{a} \approx 0.2 \mathrm{~Hz}, \eta_{\phi}$ was set to the average variance of the perturbation found around baseline (where no QRS-T complex are present), across the different beats used to build the template and $f_{0} \in\{6,7,8,9\} \mathrm{Hz}$. Figures $1(\mathrm{a})$ and $1(\mathrm{~d})$ display a typical synthetic ECG signal.

Furthermore, we produced signals varying the power of $v_{2, k}$. The signal-to-noise ratio (SNR) was modulated in the range of 0,20 and $40 \mathrm{~dB}$.

\section{Results}

For building the EKF/EKS, one need an initial estimate of $f_{c, 0}$. For this purpose we randomly selected $f_{c, 0}$ between 5 to $8 \mathrm{~Hz}$. As a primary example the output of the EKF and EKS model for FF, AA and VA estimation are shown in Figures 1(a) to 1(f). As it may be seen, results of the EKF and EKS follow accurately the FF patterns, QRS$\mathrm{T}$ and $\mathrm{f}$-wave.

To quantify the performances of the method, we employed the root mean square error (RMSE) defined as:

$$
\operatorname{RMSE}=\sqrt{\sum_{\mathrm{n}}\left(\mathrm{f}_{\mathrm{c}, \mathrm{n}}-\hat{\mathrm{f}}_{\mathrm{c}, \mathrm{n}}\right)^{2}}
$$

where $f_{c, n}$ is the original fundamental frequency and $\hat{f}_{c, n}$ is the estimated fundamental frequency.

The values of RMSE at different input SNRs, averaged over the 290 ECG segments, are plotted in Fig. 2. The simulation results show that the EKS is able to track the fundamental frequency with an error smaller than $0.22 \mathrm{~Hz}$ for all SNRs.

\section{Discussion and Conclusion}

We have validated a simple case of the previously introduced EKF/EKS model for ECG analysis during AF. By 
considering a sum of Gaussian functions for representing the VA and a single sinusoidal function with constant frequency for AA, the simple EKS structure is suitable for the situations in which less computational power is available and only short signals are available. In the proposed method, the model of VA, AA and FF are utilized as hidden state variables. These hidden-state variables are estimated as a time series through the EKF/EKS structure. Henceforth, the ECG components (VA, AA and FF) are estimated simultaneously using signal expansion over the estimated basis functions.

The designed filter/smoother was applied to synthetic ECG signals, and the results demonstrate the filter's capability in tracking the FF, VA and AA simultaneously. We found that for short recording signals, a single sinusoidal function with constant frequency is sufficient for AA modelling. Since the frequency of the sinusoidal function is constant, the proposed method is suitable for the situations in which the FF does not change in time, which is typical of short interval of observation (over long time scales the hypothesis of a constant FF starts being questionable). Mobile or e-health applications are a perfect example of a context in which the present method would prove effective.

\section{References}

[1] Stridh M, Sörnmo L. Spatiotemporal QRST cancellation techniques for analysis of atrial fibrillation. IEEE Trans Biomed Eng 2001;48:105-111.

[2] Alcaraz R, Rieta JJ. Adaptive singular value cancelation of ventricular activity in single-lead atrial fibrillation electrocardiograms. Physiol Meas 2008;29:1351-1369.

[3] Sanchez C, Millet J, Rieta JJ, Castells F, Rodenas J, RuizGranell R, Ruiz V. Packet wavelet decomposition: An approach for atrial activity extraction. In Computers in Cardiology, 2002. 2002; 33-36.

[4] Dai H, Jiang S, Li Y. Atrial activity extraction from single lead ECG recordings: Evaluation of two novel methods. Comput Biol Med 2013;43(3):176-183.

[5] Rieta J, Castells F, Sanchez C, Zarzoso V, Millet J.
Atrial activity extraction for atrial fibrillation analysis using blind source separation. IEEE Trans Biomed Eng 2004; 51(7):1176-1186.

[6] Castells F, Rieta J, Millet J, Zarzoso V. Spatiotemporal blind source separation approach to atrial activity estimation in atrial tachyarrhythmias. IEEE Trans Biomed Eng 2005;52(2):258-267.

[7] Raine D, Langley P, Murray A, Furniss SS, Bourke JP. Surface atrial frequency analysis in patients with atrial fibrillation: Assessing the effects of linear left atrial ablation. J Cardiovasc Electrophysiol 2005;16(8):838-44.

[8] Kheirati Roonizi E, Sassi R. An extended bayesian framework for atrial and ventricular activity separation in atrial fibrillation. IEEE J Biomed Health Inform 2016;submitted.

[9] McSharry PE, Clifford GD, Tarassenko L, Smith LA. A dynamic model for generating synthetic electrocardiogram signals. IEEE Trans Biomed Eng 2003;50:289-294.

[10] Kheirati Roonizi E, Sameni R. Morphological modeling of cardiac signals based on signal decomposition. Comput Biol Med 2013;43:1453-1461.

[11] Goldberger AL, Amaral LAN, Glass L, Hausdorff JM, Ivanov PC, Mark RG, Mietus JE, Moody GB, Peng CK, Stanley HE. PhysioBank, PhysioToolkit, and PhysioNet components of a new research resource for complex physiologic signals. Circulation 2000;101:e215-e220.

[12] Kheirati Roonizi E, Sassi R. A signal decomposition model-based bayesian framework for ecg components separation. IEEE Trans Signal Process 2016;64(3):665-674.

[13] Petrenas A, Marozas V, Sörnmo L, Lukosevicius A. An Echo State Neural Network for QRST Cancellation During Atrial Fibrillation. IEEE Trans Biomed Eng 2012;59:2950_ 2957.

Address for correspondence:

Ebadollah Kheirati Roonizi

Dipartimento di Informatica

Università degli Studi di Milano

Via Bramante 65, Crema, 26013, Italy

Email: ebadollah.kheirati@unimi.it 